\section{Axon-regenerating small molecules}

\section{By Lauren Martz, Staff Writer}

Researchers at the University of Miami Miller School of Medicine have identified compounds that can overcome the two main types of signals that inhibit axon growth in a damaged CNS. ${ }^{1}$ The compounds reversed the effects of neurite outgrowth inhibitors in vivo, but the molecular targets of the compounds need to be determined before they advance into humans.

The inability of axons to regenerate in damaged neurons is caused in part by inhibitory myelin-associated glycoproteins and by inhibitory proteins such as chondroitin sulfate proteoglycans (CSPGs) that are secreted by the glial scar that forms after an injury.

Previous studies have shown that upregulating cyclic adenosine monophosphate (cAMP) can help overcome myelin glycoprotein-mediated inhibition of axon regeneration. ${ }^{2}$ Also, inhibitors of protein kinase $\mathrm{C}(\mathrm{PKC})$ and epidermal growth factor receptor (EGFR) have been associated with regeneration of neurons affected by CSPGmediated inhibition. . $^{3-5}$

Now, a paper published in The Journal of Neuroscience describes axon-regenerating compounds that reverse both myelin- and glial scarinduced growth inhibition.

The University of Miami team used a combinatorial library of triazinebased compounds with unknown targets to screen for molecules that allowed damaged neurons to grow.

In embryonic neurons on growth-inhibiting myelin substrates, four compounds reversed growth inhibition as efficiently as cAMP. In a second screen of neurons on a mixture of CSPGs, the same four compounds reversed neurite growth inhibition with potencies similar to those for PKC inhibitors.

The results in the embryonic neurons were replicated in mature neurons, which have a decreased ability to grow and proliferate, and in other neuronal populations including spinal cord neurons and cortical neurons.

The team then tested the compounds on a complex cellular substrate that recapitulated the diverse inhibitory signals of a glial scar in vivo. In this scar model, the most effective of the four compounds almost doubled the number of neurons that grew neurites compared with no treatment. The molecule also increased neurite length by about $50 \%$.
In mice, the compounds increased injured axon growth compared with saline control.

The compounds had no effect on control neurons that were not exposed to either myelin or CSPGs. That result suggested that the molecules actually reversed the inhibition of neuron growth rather than simply promoting neuron growth.

In culture, the group showed that the lead compound did not act on cAMP, PKC or EGFR, suggesting that it likely reversed growth inhibition via a new mechanism.

Although the researchers haven't uncovered the specific pathway, they did show that the compound stimulated microtubule growth, suggesting it might act on neurite outgrowth through a microtubule-generating mechanism.

"The compounds appear to be working through a novel mechanism unlike those targeted by compounds currently in therapeutic development," said team leader John Bixby, professor of pharmacology and neuroscience and director of the graduate program in biomedical sciences at the university's Miller School of Medicine.

Novartis AG's preclinical candidate ATI355 targets reticulon 4 (RTN4; NOGO-A; NOGO), a component of myelin, whereas Acorda Therapeutics Inc.'s chondroitinase ABCI, which is in preclinical development to treat spinal cord injury (SCI), targets the CSPGs produced by the glial scar.

According to Shuxin $\mathrm{Li}$, assistant professor in the Department of Neurology at The University of Texas Southwestern Medical Center at Dallas, one of the main outstanding questions is whether the compounds "promote functional regeneration in animal models, such as spinal cord injury."

"What they need to do after they identify or develop their lead compound is to study it in animal models of spinal cord and brain injury," agreed Anthony Caggiano, VP of preclinical development at Acorda Therapeutics. "They will need to find evidence of both regeneration and functional benefit in these animals."

\section{Target identification}

Bixby said his group is screening a number of related compounds in an assay similar to the one used in the Journal of Neuroscience paper; those compounds will then be developed further in SAR studies. Future studies to determine functional regeneration are being planned.

"The most important issues are to identify a clear mechanism of action, to obtain toxicology and pharmacokinetic profiles of the individual compounds and to test at least one lead compound in other animal models of CNS injury," said Bixby. "The lack of a clear primary target can make people uneasy."

Indeed, Li noted that triazine compounds are commonly used as the basis for herbicides. As a result, he said, "it is essential to determine 


\section{ANALYSIS}

whether the compounds react with multiple molecules and also interrupt other signaling pathways in the body."

Jerry Silver, professor in the Department of Neurosciences at Case Western Reserve University, wanted to know whether the compounds from Bixby's group are able to cross the blood brain barrier (BBB). The ability to do so would be ideal, he said, because it would avoid the need to deliver therapeutics directly into the CNS through a needle in the brain or spinal cord.

Acorda's Caggiano was sanguine about the potential of the University of Miami molecules to cross the BBB. "The researchers chose compounds that follow Lipinski's rules, which cover drug characteristics including size and hydrophobicity. Because the chosen compounds do follow these rules, it is possible that they will cross the BBB and can be systemically delivered," he said.

Even if the molecules are able to cross the BBB, Silver thinks potency could be an issue. "In my opinion, the novelty of the discovery and the mechanism of action are more interesting than the amount of regeneration stimulated in vivo. The findings are relatively modest."

Thus, he said, Bixby's team needs to "identify other related compounds and try other concentrations of the compounds identified to get stronger regeneration data."

\section{TARGETS \& MECHANISMS}

Silver did say that because the compounds described in the paper work through a new mechanism, they could represent "a brand-new strategy that could have synergy with existing strategies."

Bixby said the University of Miami has filed a patent application covering the compounds and the IP is available for licensing.

Martz, L. SciBX 3(15); doi:10.1038/scibx.2010.457

Published online April 15, 2010

\section{REFERENCES}

1. Usher, L. et al. J. Neurosci.; published online March 31, 2010; doi:10.1523/JNEUROSCI.0302-10.2010

Contact: John L. Bixby, University of Miami Miller School of Medicine, Miami, Fla.

e-mail: jbixby@med.miami.edu

2. Lehmann, M. et al. J. Neurosci. 19, 7537-7547 (1999)

3. Sivasankaran, R. et al. Nat. Neurosci. 7, 261-268 (2004)

4. Koprivica, V. et al. Science 310, 106-110 (2005)

5. Ahmed, Z. et al. Neurobiol. Dis. 36, 142-150 (2009)

COMPANIES AND INSTITUTIONS MENTIONED

Acorda Therapeutics Inc. (NASDAQ:ACOR), Hawthorne, N.Y. Case Western Reserve University, Cleveland, Ohio Novartis AG (NYSE:NVS; SIX:NOVN), Basel, Switzerland University of Miami Miller School of Medicine, Miami, Fla. The University of Texas Southwestern Medical Center at Dallas, Dallas, Texas 